\title{
Image reconstruction via phase conjugation in amorphous chalcogenide thin films
}

\author{
M. Fernández-Guasti ${ }^{1}$, R.F. Alonso-Pinzón, E. Haro-Poniatowski ${ }^{*, 2}$ \\ Departamento de Física, Universidad Autónoma Metropolitana Iztapalapa, Apartado Postal 55-532, México 09340 D.F., México
}

Received 7 November 2002; received in revised form 24 February 2003; accepted 24 March 2003

\begin{abstract}
The restoration of an image is demonstrated using phase conjugation (PC) in amorphous $\mathrm{Ge}_{x} \mathrm{Se}_{1-x}$ thin films. An unconventional degenerate four-wave mixing scheme is used, where no external counter propagating pump beam is required. Various film configurations are reported that support the proposal that this wave is generated near the front surface of the nonlinear material. The PC signal behavior as a function of time is presented and described in terms of a simple model.
\end{abstract}

(C) 2003 Elsevier Science B.V. All rights reserved.

\section{Introduction}

Optical memory effects in amorphous semiconductors films were demonstrated a few decades ago [1]. In particular amorphous chalcogenide thin films have been actively investigated as holographic recording media [2-4]. An important feature is that in many cases the physical processes involved in the writing procedure are reversible [5]. Diffraction gratings formation mechanisms have also been discussed in these materials [4,6]. Light-

\footnotetext{
${ }^{*}$ Corresponding author. Fax: +34-91-564-55-57.

E-mail addresses: haro@io.cfmac.csic.es, haro@xanum. uam.mx (E. Haro-Poniatowski).

${ }^{1}$ On sabbatical leave at Instituto Nacional de Astrofísica, Óptica y Electrónica, Apdo. Postal 51 y 216, 72000 Puebla, Pue., México.

${ }^{2}$ On sabbatical leave at Instituto de Óptica, CSIC, Serrano 121, 28006 Madrid, Spain.
}

induced modifications of bonding defects have been proposed in order to understand the underlying processes occurring under laser irradiation in chalcogenide glasses [7]. Several types of defects have been suggested, some of them producing states below the absorption edge. In addition chalcogenide glasses in thin film and bulk forms exhibit other interesting nonlinear optical properties. Photodarkening and photobleaching effects have been reported in the past [8]. More recently optical bistability and optical phase conjugation (PC) in these materials have been investigated [912]. These effects generally occur at low irradiation intensities with sub-band gap photons making these materials interesting for technological applications in opto-electronics.

The chalcogenide glasses are by definition those including $\mathrm{Se}, \mathrm{Te}, \mathrm{S}$, as one of their constituents at least. Examples in which one or more of the abovementioned nonlinear effects have been observed 
are $\mathrm{Se}, \mathrm{As}_{2} \mathrm{~S}_{3}, \mathrm{Ge}_{x} \mathrm{Se}_{1-x}, \mathrm{Se}, \mathrm{Se}_{x} \mathrm{Te}_{1-x}$ as well as ternary and quaternary compounds such as As$\mathrm{Ge}-\mathrm{Te}$, Te-Ge-Sb-S, and As-Se-S-Ge $[2,3,5,10]$. The observed properties involve photo-induced structural effects occurring through bond breaking or bond reorientation [7,13]. Purely phenomenological models have also been proposed [12].

In previous work we have analyzed grating formation in different systems and in particular in pure selenium [10,11]. It was first supposed that the formation of the fringes in pure amorphous selenium was due to a chemical reaction process or to the sublimation of $\mathrm{Se}$ in the interfringes. In order to exclude these possibilities a layer of $\mathrm{GeO}_{2}$ was deposited covering the selenium thin film. A diffraction grating was equally formed in the Se film despite this protective layer thus excluding a sublimation process $[10,11]$. Therefore, the photoinduced structural effects seem to be directly responsible for the formation of the diffraction grating.

The capability of image correction via PC in amorphous $\mathrm{As}_{2} \mathrm{~S}_{3}$ has been previously reported using a simple mask [12]. In the case of selenium films, we have investigated the PC signal behavior as a function of the thickness of the sample [14]. $\mathrm{PC}$ was observed even in very thick samples, where reflection at the back surface is negligible due to the strong absorption of the material. Furthermore, preliminary experiments in a bulk sample of amorphous $\mathrm{GeSe}_{2}$, with $1.5 \mathrm{~mm}$ thickness confirmed these observations. The main result is that in order to explain this behavior with a degenerate four-wave mixing scheme (D4WM) a counter propagating pump beam generated near the surface of the film has to be assumed leading to a new D4WM configuration.

In the present work we investigate the reconstruction of an image via PC in amorphous chalcogenide $\mathrm{Ge}_{x} \mathrm{Se}_{1-x}$ thin films. Concerning the structural reorientation and pumping scheme, the PC signal has been obtained for different filmsubstrate configurations. On the one hand, these experiments support the light-induced reordering mechanism as responsible for the grating formation. On the other hand, they sustain the proposal that a novel D4WM process occurs, where the counter propagating pump is generated at the surface of the nonlinear material.

\section{Experimental setup}

The setup was placed on a stable holographic table due to the slow response time of the nonlinear recording materials. Different $\mathrm{Ge}_{x} \mathrm{Se}_{100-x}$ and $\mathrm{Te}_{x} \mathrm{Se}_{100-x}$ chalcogenide films with constant " $x$ " between 1.5 and 20 were used as recording media. Thin films with $2.5 \mu \mathrm{m}$ thickness were evaporated onto Corning 7059 glass substrates. The films were exposed in air at room temperature.

A $35 \mathrm{~mW} \mathrm{HeNe}$ laser beam was split into a pump and probe beams with relative intensities of 10:1.7 as shown in Fig. 1. The films were posi-

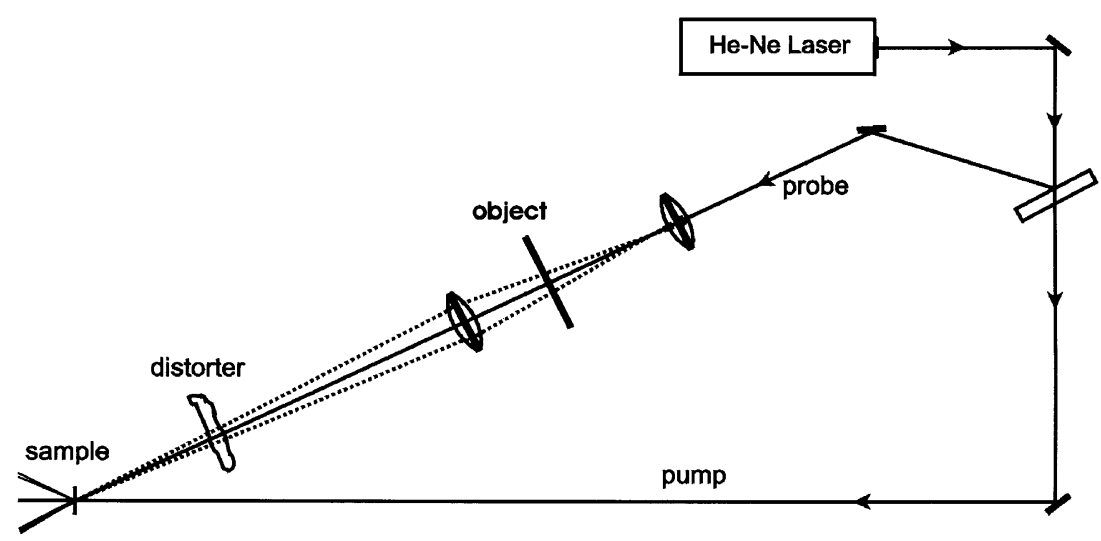

Fig. 1. Experimental setup used to observe phase conjugation in amorphous chalcogenide thin films. 
tioned perpendicularly to the pump beam. The pump beam diameter at the film was $120 \mu \mathrm{m}$. In order to illuminate an object slide of $5.5 \times 1.8 \mathrm{~cm}$, the probe beam was expanded with a bi-convex lens. The slide was adequately illuminated when placed at $35 \mathrm{~cm}$ from the lens. The object to be conjugated was the word "Saasil" which means light in Mayan language. It was imprinted in a microscope slide with auto-adhesive letters. The diverging beam containing the object information was then focused back on the film insuring an adequate overlap with the pump beam. The angle between pump and probe beam was $10^{\circ}$. The probe beam converging onto the recording material was distorted by an aberrating element placed at $15 \mathrm{~cm}$ before reaching the recording plane. Two Petri dishes were used for this purpose. The angle of acceptance of the phase conjugate mirror was limited by the area covered by the pump beam.

The image was permanently recorded onto the nonlinear film after a few minutes exposure. Once the image was recorded, photographs of the object reconstructed by a PCM and a conventional mirror were taken with a reflex camera without its lens attached. The reference image produced by the object was photographed just after the object plane under the illumination of the probe beam. The conjugated image was reconstructed with the pump beam and photographed at the object plane; i.e., the photographic film was at the same position of the original object but facing towards the conjugated beam now containing the recorded image. The image reflected on a conventional mirror was taken with the probe beam illuminating the object and then falling onto a mirror placed at the phase conjugator plane but perpendicular to the probe beam. The reflected image was deviated via a beam splitter and photographed at a distance equal to the object to mirror path.

Phase conjugate signals with efficiencies $\left(I_{\text {pc }} / I_{\text {probe }}\right) \times 100$ between $0.17 \%$ and $0.74 \%$ were obtained for films with selenium content " $x$ " between 20 and 1.5. These efficiencies are much lower than the $18 \%$ reported before [11], due to the lower power density incident on the film. However, in these series of experiments a compromise was adopted in order to cover a large film area while achieving efficiencies that allowed comfortable recording of the images. The typical power of the phase conjugate signal was between 5.8 and $25 \times 10^{-6} \mathrm{~W}$. The temporal behavior of the PC signal was monitored with a beam splitter placed in the optical path of the retro propagated beam and steered into a photo detector. A polished bulk $\mathrm{GeSe}_{2}$ glass of $1.5 \mathrm{~mm}$ thickness was also used as a PC mirror.

\section{Results and discussion}

Comparison of the original object (Fig. 2(a)) and the phase conjugate image after passing through the aberrator (Fig. 2(b)) reveals that the lettering is legible in the latter image. The image reflected on the conventional mirror is shown in Fig. 2(c). The severe obliteration produced in this image makes the original object completely unrecognizable. These results unambiguously show that $\mathrm{Ge}_{x} \mathrm{Se}_{100-x}$ thin films with the above-mentioned pumping scheme act as PC mirrors with the corresponding aberration correction properties.

Nonetheless, the corrected image presents imperfections, in particular, vertical fuzzy lines stemming from the aberrator. These lines are clearly observed in the glass of the Petri dishes. One of them was rotated by $90^{\circ}$ to confirm that this was the origin of the pattern as shown in Fig. 2(d), where a set of perpendicular fuzzy lines are observed in the background. Zones of the dishes with severe deformation deviate part of the probe beam into regions of the nonlinear film where the pump is not present or even move them away from the film. These high-spatial frequency components are obviously no longer conjugated and limit the quality of the image reconstruction.

The recording material was usually placed with the film facing towards the pump and probe beams. However, in order to investigate the role of the film-air and film-substrate interfaces, the substrate was placed facing towards the incident beams. The PC signal intensity was unaltered in either configuration. Since in the film-substrate interface sublimation of selenium cannot occur this mechanism has to be ruled out, thus strengthening the view that the photostructural transformation is responsible for the grating formation [11]. 

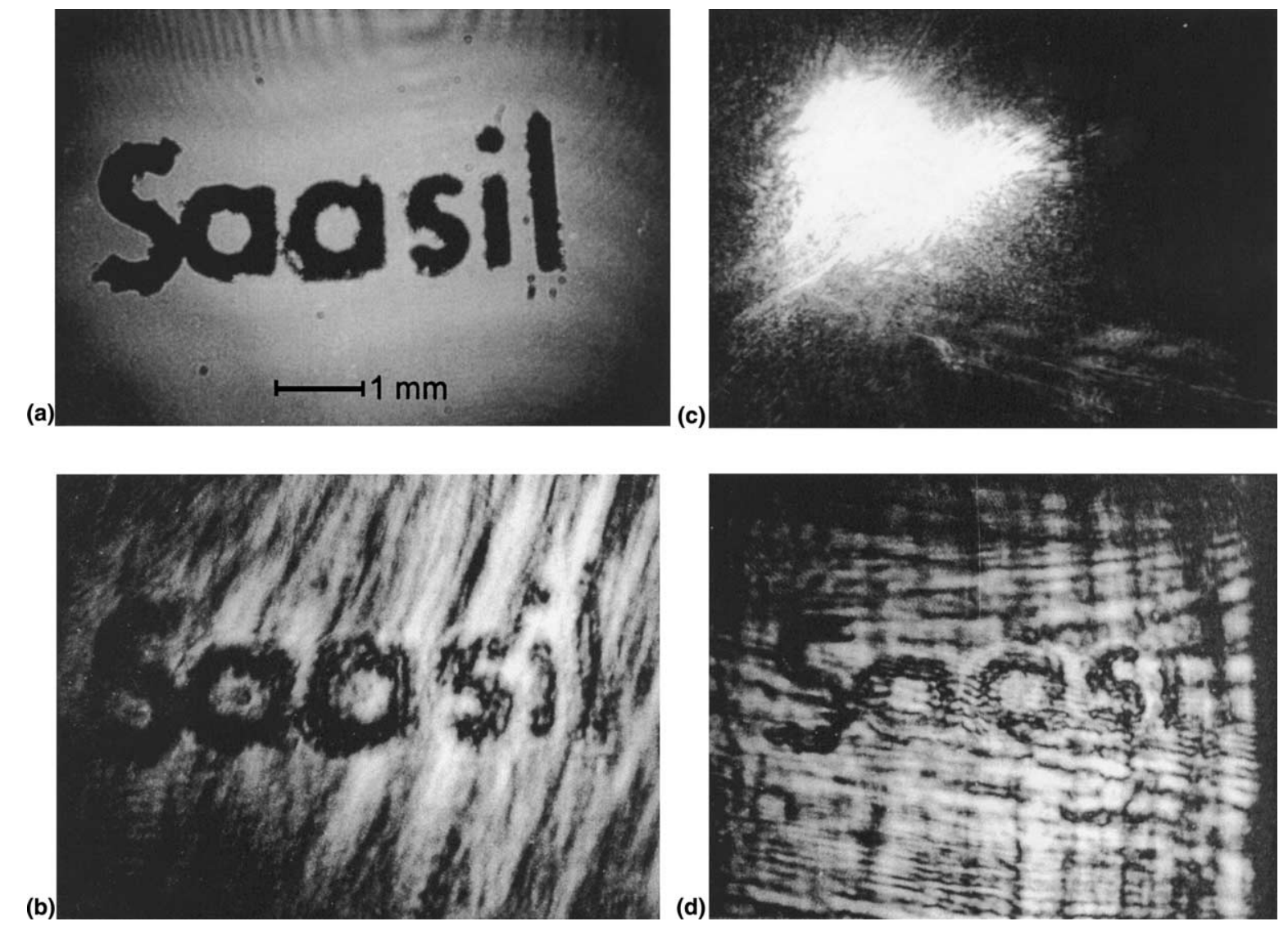

Fig. 2. (a) Original probe beam image; (b) phase conjugate corrected image; (c) distorted image obtained from conventional reflection and (d) phase conjugate image with crossed Petri dishes as aberration element.

The pump beam experiences strong absorption as it propagates through the $\mathrm{Ge}_{x} \mathrm{Se}_{100-x}$ film. Placing a high-reflectivity mirror after the substrate in order to provide an externally counter propagating pump had no effect on the PC signal intensity. In order to evaluate the contribution of the film-substrate interface to the counter propagating pump, a $\operatorname{GeSe}_{2}(x=33)$ bulk sample was used as a recording medium. The bulk was polished so that specular reflection was obtained from the front surface. The back surface was left unpolished and no specular reflection could arise from it. Furthermore, an absorption coefficient of the order of $10^{3} \mathrm{~cm}^{-1}$ insures that any reflection coming from the back surface is at least 1000 times less intense. A phase conjugate signal was nevertheless detected albeit less intense than in the film case. It is therefore reasonable to consider that the counter propagating pump must be generated in the vicinity of the front interface in either the film or the bulk material. In the latter case, this claim is reinforced by the fact that there is no other interface, where the counter pump could be generated. The lower PC efficiency in the bulk compared with the thin film is most likely due to the low selenium content of the bulk sample. On the other hand, when the film or the bulk was rotated so that the pump was no longer incident at normal incidence no PC signal was detected. This experience is in accordance with a reflected pump wave that is no longer conjugate of the incident one. Further evidence in support of the front surface pump reflection comes from previous experiments, where the PC intensity was monitored as a function of 
the thickness of the film [14]. In those results, the PC intensity reached a maximum intensity at approximately $15,000 \AA$ and no longer increased for thicker samples. The penetration of the reflected wave into the second medium may be explained in terms of the extinction theorem. According to this approach, the Ewald Oseen dipole field oscillates coherently in order to produce the transmitted and reflected wave. The penetration depth where the dipole field contributes to the reflected wave is in this case limited by the absorption coefficient of the material. Analytical solutions to this problem are in good agreement with the variable thickness experimental results [14]. Therefore, the proposal that the counter propagating pump is generated in the vicinity of the front surface air-film interface is consistent with the experimental results as well as with the theoretical notions provided by the extinction theorem.

The typical temporal behavior of the PC signal is shown in Fig. 3. The intensity increases drastically during the first 2 min of exposure and reaches a saturation value thereafter. Let us recall the PC and spatial grating formation in amorphous chalcogenide thin films reported in a previous work [11]. The main result was that the surface relief of the grating alone does not seem enough to explain the experimental results and volume effects have to be considered. Volume refractive index changes can occur in chalcogenide glasses through structural reordering at irradiation energies near or

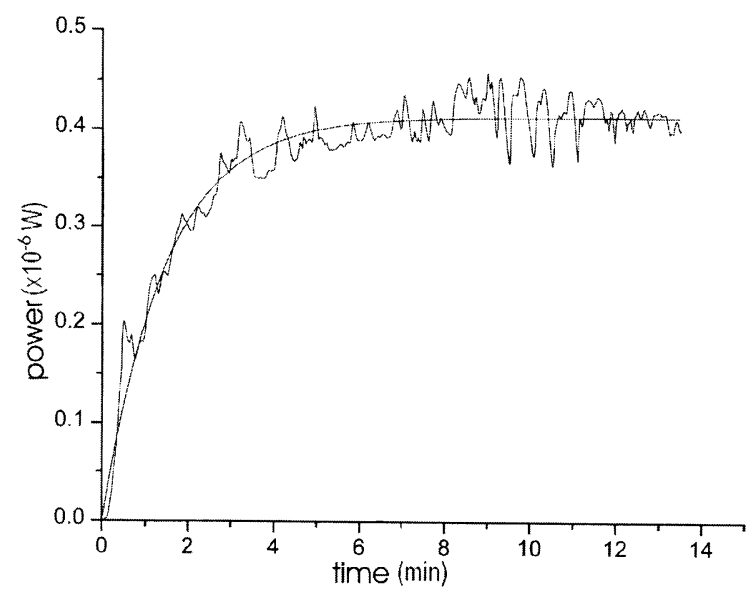

Fig. 3. Phase conjugate signal intensity versus time. below the absorption edge [7]. Therefore, in addition to the relief present at the surface, structural rearrangements may be induced within the film at high-intensity regions. A second grating is then generated that consists of stripes of film with different refractive index. This second grating is mainly responsible for the observed PC signal $[10,11]$. The material that is transformed by light irradiation can be described analogously to a photo-crystallization process [15] by an expression of the type [16]

$I=I_{\mathrm{s}}\left(1-\mathrm{e}^{-\alpha t}\right)$.

The smooth curve shown in Fig. 3 shows an attempt to fit the experimental data with $I_{\mathrm{s}}=0.41 \times 10^{-6} \mathrm{~W}$ and $\alpha=0.68 \mathrm{~s}^{-1} ; I_{\mathrm{s}}$ represents the saturation intensity level and $\alpha$ is a measure of the growth rate. The agreement between this simple model and the experimental curve suggests that the intensity of the PC signal is proportional to the photo-transformed volume of material.

\section{Conclusions}

The capability of image restoration via D4WM in germanium selenium amorphous thin films has been experimentally demonstrated. These results are likely to be reproduced in other chalcogenide systems and are consistent with a photostructural transformation model. The counter propagating pump beam need not be externally injected and it seems plausible that it is generated at the air-film interface.

\section{References}

[1] S.R. Ovshinsky, Phys. Rev. Lett. 21 (1968) 1450.

[2] Sakae Zembutsu, Yoshio Toyoshima, Takeo Igo, Haruo Nagai, Appl. Opt. 14 (1975) 3073.

[3] A. Singh, L. Song, R.A. Lessard, Appl. Opt. 26 (1987) 2474.

[4] J.M. Ballesteros, R. Hernández, J.M. Herreros, C.N. Afonso, A.K. Petford-Long, R.C. Doole, Appl. Phys. A 65 (1997) 463.

[5] Seung Goi Lee, Sang Soo Lee, Appl. Opt. 25 (1986) 4512.

[6] T.W. Hou, M.S. Chang, Appl. Opt. 18 (1979) 1753.

[7] S.R. Elliott, J. Non-Cryst. Solids 81 (1986) 71. 
[8] N. Kumagai, J. Shirafuji, Y. Inuishi, J. Phys. Soc. Jpn. 42 (1977) 1261.

[9] E. Haro-Poniatowski, M. Fernández-Guasti, E.R. Mendez, M. Balkanski, Opt. Commun. 70 (1989) 70.

[10] E. Haro-Poniatowski, M. Fernández-Guasti, S. CamachoLópez, Opt. Lett. 17 (1992) 252.

[11] E. Haro-Poniatowski, M. Fernández-Guasti, S. CamachoLópez, F. Ruiz, Physica A 207 (1994) 329.

[12] J.H. Kwon, C.H. Kwak, S.S. Lee, Opt. Lett. 10 (1985) 568.
[13] J.E. Griffiths, G.P. Espinosa, J.P. Remeika, J.C. Phillips, Phys. Rev. B 25 (1982) 1272.

[14] E. Haro-Poniatowski, M. Fernández-Guasti, S. CamachoLópez, A. Gil-Villegas, J. González-Hernández, Opt. Commun. 119 (1995) 154.

[15] E. Haro-Poniatowski, C. Julien, B. Pecquenard, J. Livage, M.A. Camacho-López, J. Mater. Res. 13 (1998) 1033.

[16] J.W. Christian, in: The Theory of Transformations in Metals and Alloys. Equilibrium and General Kinetic Theory, Pergamon Press, New York, 1975, p. 19 (Part I). 